\title{
Structural Insights into Putative Molybdenum Cofactor Biosynthesis Protein C (MoaC2) from Mycobacterium tuberculosis H37Rv
}

\author{
Vijay Kumar Srivastava", Shubra Srivastava', Ashish Arora*, J. Venkatesh Pratap* \\ Molecular and Structural Biology Division, CSIR-Central Drug Research Institute, Mahatma Gandhi Marg, Lucknow, India
}

\begin{abstract}
The Molybdenum cofactor (Moco) biosynthesis pathway is an evolutionary conserved pathway seen in almost all eukaryotes including the pathogenic species Mycobacterium tuberculosis. This pathway comprises of several novel reactions which include the initial formation of precursor Z from guanosine triphosphate (GTP), catalysed by two enzymes MoaA and MoaC. Although Moco biosynthesis is well understood, the first step is still not clear. In M. tuberculosis H37Rv, three orthologous genes of MoaC have been annotated: moaC1 (Rv3111), moaC2 (Rv0864) and moaC3 (Rv3324c). Rv0864 (MoaC2) is a $17.5 \mathrm{kDa}$ protein and is reported to be down-regulated by $\sim 3$ times in the nutrient starvation model for Mycobacterium tuberculosis. The crystal structure of Moco-biosynthesis protein MoaC2 from Mycobacterium tuberculosis (2.20 $\AA$ resolution, space group $P 2{ }_{1} 3$ ) has been determined. Based on a comparative analysis of structures of homologous proteins, conserved residues were identified and are implicated in structural and functional roles. Molecular docking studies with probable ligands carried out in order to identify its ligand, suggests that pteridinebenzomonophosphate as the most likely ligand. Sequence based interaction study identified MoaA1 to interact with MoaC2. A homology model of MoaA1 was then complexed with MoaC2 and protein-protein interactions are also discussed.
\end{abstract}

Citation: Srivastava VK, Srivastava S, Arora A, Pratap JV (2013) Structural Insights into Putative Molybdenum Cofactor Biosynthesis Protein C (MoaC2) from Mycobacterium tuberculosis H37Rv. PLoS ONE 8(3): e58333. doi:10.1371/journal.pone.0058333

Editor: Narayanaswamy Srinivasan, Indian Institute of Science, India

Received October 24, 2012; Accepted February 1, 2013; Published March 19, 2013

Copyright: (c) 2013 Srivastava et al. This is an open-access article distributed under the terms of the Creative Commons Attribution License, which permits unrestricted use, distribution, and reproduction in any medium, provided the original author and source are credited.

Funding: This work was supported by a Council of Scientific and Industrial Research, India (CSIR) network grant NWP0038 to AA. Computational support was from CSIR grant CMM0017 and DST grant to JVP. The funders had no role in study design, data collection and analysis, decision to publish or preparation of the manuscript

Competing Interests: The authors have declared that no competing interests exist.

*E-mail: ashish_arora@cdri.res.in (AA); jvpratap@cdri.res.in (JVP)

9 These authors contributed equally to this work.

\section{Introduction}

Rv0864 (MoaC2) from Mycobacterium tuberculosis is one of the enzymes in the molybdenum cofactor (Moco) biosynthesis pathway. Together with MoaA, MoaC is involved in the conversion of guanosine triphosphate (GTP) to precursor Z, the first step in Moco synthesis [1,2]. Details of the Moco biosynthesis pathway, including the types of molybdenum cofactors and genetic deficiencies caused due to Moco biosynthetic enzymes, have been discussed in Srivastava et al., 2012 [3]. MoaA belongs to the Sadenosylmethionine (SAM)-dependent radical enzyme superfamily, members of which catalyse the formation of protein or substrate radicals by reductive cleavage of SAM by a $[4 \mathrm{Fe}-4 \mathrm{~S}]$ cluster $[4,5,6]$. Although moco biosynthesis is well understood, its first step, the synthesis of precursor Z from GTP, is still not clear. It is thought that the GTP molecule first binds to MoaA and an intermediate compound (formamidopyrimidine-type; FPT) is generated which is subsequently used by MoaC. MoaC catalyzes the release of pyrophosphate from FPT and the formation of the cyclic phosphate of precursor Z [7]. Kanaujia et al., (2010) [8] proposed that the formation of precursor $\mathrm{Z}$ can occur in two possible pathways: in the first path where FPT (formamidopyrimidine-type) is the substrate of MoaC [7] and precursor Z is formed either via the formation of intermediate compound $\mathrm{E}$ (formamido-type) or PBM (pteridinebenzomonophosphate), and in the second case, compound PBT (pteridinebenzotriphosphate) may play the role of the substrate of MoaC which is formed when the ring formation of FPT molecule is completed. In this pathway, $\mathrm{PBM}$ is the intermediate formed before precursor $\mathrm{Z}$ (Figure 1).

At present, it is also unclear whether MoaA and MoaC act independently of each other or form a protein-protein complex. In humans, the molybdenum cofactor synthesis step 1 (MOCS1) locus has been reported to produce two enzymes (MOCS1A and MOCS1B) from non-overlapping ORFs within a bicistronic transcript [9]. The functional characterization of the human proteins MOCS1A and MOCS1B as well as the MOCS1AMOCS1B fusion protein that catalyze the formation of precursor $\mathrm{Z}$ have been identified [9,10,11]. MOCS1A (MoaAs) from eubacteria and eukaryotes contain a functionally important double Gly motif at the C-terminus [12]. A likely function of this double glycine motif in MOCS1A might be the interaction with MOCS1B, forming a stable MOCS1A-MOCS1B protein complex or a transient complex during catalysis, as in the MoaD-MoaE and MoeB-MoaD protein complexes [13,14]. The C-terminal loop of MOCS1A might interact with the active site of MOCS1B, which is formed by two monomers in the homologous MoaC protein from E. coli [15].

In the Betts et al., 2002 [16] starvation model, mimicking Mtb, many genes appear to be down-regulated, including those that are 

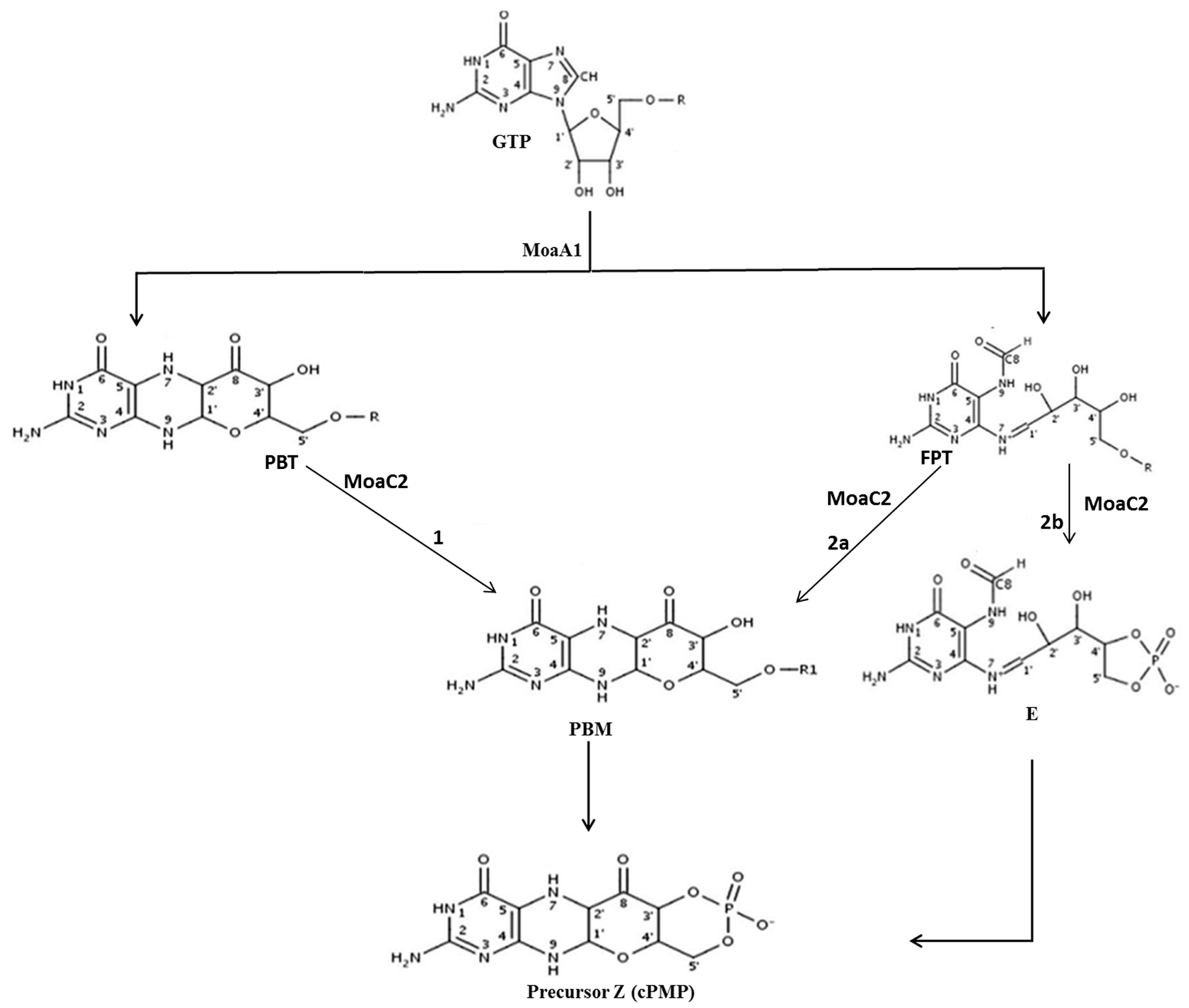

Figure 1. Schematic representation of the possible mechanisms proposed for the first step of the Moco biosynthesis pathway involving two probable substrate molecules FPT and PBT for MoaC. R and R1 denote triphosphate and monophosphate groups, respectively [8].

doi:10.1371/journal.pone.0058333.g001

involved in energy metabolism, lipid biosynthesis, cell division, and transcription. In contrast, starvation induces a number of stress response and virulence factors that may be important for adaptation to the host environment. In this model MoaC2 is reported to be down-regulated by $\sim 3$ times after 4 hour starvation. Precursor $\mathrm{Z}$ is one of the most stable intermediates in the Moco pathway having a half-life of several hours at low $\mathrm{pH}$ [17]. In addition to these orthologous gene moaCl (Rv3111) is necessary for intermediary metabolism. In primate model, mutation in this particular gene of Mycobacterium tuberculosis causes attenuation therefore the mechanism of Moco pthaway is necessary to understand which is still not clear [18].

Here we report the crystal structure of MoaC2 from Mycobacterium tuberculosis $H 37 R v$. The cloning, expression, purification and crystallization were already discussed in Srivastava et al., 2012 [3]. As our attempts to crystallize the protein with guanosine triphosphate (GTP) or other ligands have been unsuccessful, computational docking studies were initiated with the probable ligands from the pathways proposed by Kanaujia et al., 2010 [8] in the formation of precursor Z. In addition sequence based interaction study was done to know if MoaC2 and MoaA interact with each other and further protein-protein docking was done with identified interacting partner.

\section{Results and Discussion}

\section{The Structure of MoaC2}

The MoaC2 monomer has a common $\alpha+\beta$ protein fold that is composed of a four stranded anti-parallel $\beta$-sheet and three helices, $\alpha 1, \alpha 2$ and $\alpha 3$ located underneath the $\beta$-sheet (Figure 2 a). The fold of MoaC belongs to the ferredoxin like family $(\beta \alpha \beta \beta \alpha \beta)$ with one additional helix $(\beta \alpha \alpha \beta \beta \alpha \beta)$ not typically present in a prototypical ferredoxin-like molecule. The additional helix $(\alpha 1)$ which is not typically present in ferredoxin like fold, may impart functional role. The asymmetric unit of Rv0864 (MoaC2) contains a dimer and three such dimers related by crystallographic 
symmetry associate to form a hexamer, which is the biological assembly. The two independent subunits in the structure superpose onto each other with a root mean square deviation (r.m.s.d) of $0.35 \AA$ for $120 \mathrm{C} \alpha$ atoms. Closer examination of the superposition reveals conformational differences in the additional helix $\alpha 1$, wherein the r.m.s.d between $\alpha 1$ region of subunit $A$ and $\mathrm{B}$ is $0.46 \AA$. Upon dimerization, the 4 -stranded anti parallel beta sheets of both monomers associate to form a contiguous 8 stranded sheet. The $\alpha 3$ helix is present at the dimer interface of MoaC2, $\alpha 2$ helix, loop region residues 83-89 (LIPLCHQ) and Leu122 and Ile148 are mainly involved in the dimerization of the protein molecule. The surface areas buried at the intra-dimeric and interdimeric interfaces in the hexamer are given in Table 1 . The intradimeric interface area is greater than the other interface. Also, while 14 hydrogen bonds stabilize this interface, while eight hydrogen bonds are observed in the other interface. From the surface area calculations and number of inter subunit hydrogen bonds at the interfaces, it can be speculated that MoaC2 exists as a trimer of dimers wherein the dimer counterparts are held together by hydrophobic and hydrophilic interaction. During oligomerization, a total of $21 \%$ and $42 \%$ area of monomeric units was buried upon dimerization and hexamer formation. The solvation free energy gain upon formation of dimer and hexamer assembly, calculated using the PISA webserver [19] are $-25 \mathrm{kcal} \mathrm{mol}^{-1}$ and $-106.9 \mathrm{kcal} \mathrm{mol}^{-1}$ which suggests that hexamer assembly is more stable.

The structure of MoaC2 was solved in its native form, without any ligand. The putative binding sites, as identified from a superposition with the homologous T. thermophilus structure (PDB ID 3JQM) [8] are shown as stick in monomer, dimer and asterisk in hexamer (Figure $2 \mathrm{a}, \mathrm{b}, \mathrm{c}$ ).

\section{Comparison of Rv0864 (MoaC2) with its homologues from other sources}

Structural information on MoaC is available from apo crystal structures from Escherichia coli (EcMoaC; PDB entry lekr) [5], Pyrococcus horikoshii (PhMoaC; PDB entry 2ekn, Sulfolobus tokodaii (StMoaC; PDB entry 2ohd [20], Geobacillus kaustophilus (GkMoaC; PDB entry 2eey and GTP bound from Thermus thermophilus (TtMoaC PDB entry 3JQM) [8] are available.

A structural based sequence alignment of Rv0864 with E.coli, T.thermophilus, G. kaustophilus, P.horikoshii and S.tokodaii is shown in Figure 3. The overall sequence identity of Rv0864 with all other MoaC proteins is $17 \%$ though the pairwise sequence identity of these proteins with Rv0864 are higher ( $\sim 4 \%$ with E.coli, T.thermophilus, G.kaustophilus, P.horikoshii and with $\sim 35 \%$ with S.tokodain) .

It also shows that 31 of 167 residues are conserved among the species. These conserved residues play an important functional or structural role. The Ala69, Gly73, Ala76 and Lys78 present on the $\alpha 2$ helix play a significant role in the oligomerization of protein the protein molecule. Lys78 which is conserved in all MoaC proteins is located in the $\alpha 2$ helix of the adjacent dimer forms three strong hydrogen bonds through its NZ (Average distance $2.8 \AA$ ) to the main chain carbonyl group of residues Ser81, Pro85 and Cys87, with an average distance of $\sim 2.8 \AA$ resulting in an approximately three fold symmetrical arrangement.

The tertiary structure of Rv0864 (MoaC2) is similar with T.thermophilus, E.coli, G.kaustophilus, P.horikoshii and S.tokodaii with root mean square deviation of $1.45,0.96,2.7,1.4$ and $0.98 \AA$ respectively (Figure 4). The maximum deviation is in the $\alpha 1$ region (Table S1). Each dimer of Rv0864 is stabilized by 14 hydrogen bonds as compared to eleven in T.thermophilus Moac [8] and eight hydrogen bonds in the E.coli MoaC [15].
In order to know the putative ligand binding site, the coordinates of Rv0864 (MoaC2) was superposed on T. thermophilus [8] coordinates with a root mean square deviation of $1.45 \AA$. The GTP forms hydrogen bond with Lys62, Asp137, Lys140 of A chain with distance $3.4 \AA, 2.1 \AA, 3.1 \AA$ and His88 of $\mathrm{B}$ chain with distance $2.6 \AA$, which are in qualitative agreement with the $T$. thermophilus structure. Additionally, three water molecules form four hydrogen bonds with GTP, two of them with an oxygen of the third phosphate $\mathrm{O} 3 \mathrm{G}$ with distances of $2.7 \AA$, $2.3 \AA$, and the other two with $\mathrm{O} 1 \mathrm{G}$ and $\mathrm{O} 2 \mathrm{G}$ with distances of $2.4 \AA$ and $2.5 \AA$ respectively. These water mediated interactions are not observed in T. thermophilus structure [8] (Figure 5).

The protein MoaC2 is mainly exist in a hexameric form which, however, easily dissociates into its dimer in solution, while the functional oligomeric state is unknown [21]. We analyzed the oligomerization states of all available crystal structures of MoaC using the PISA web server. The result suggests that EcMoaC, TtMoaC, StMoaC, PhMoaC and GkMoaC are predicted to be stable in dimeric, trimeric or hexameric state, with the hexameric state being the most stable. It is interesting to note that the six MoaC structures in the PDB (including Rv0864) differ in their crystallization conditions, space groups and asymmetric content (monomer to a nonamer), yet exhibit this hexameric assembly.

\section{Result of Docking}

As mentioned earlier, the first step in Moco biosynthesis, the formation of precursor $\mathrm{Z}$ from a guanosine derivative (GTP) is still unclear. It is reported that MoaA generates an intermediate compound from the guanosine derivative and subsequently MoaC acts on it to form precursor Z [7]. Our attempts to crystallize MoaC with GTP (either by co-crystallization or by soaking) have been unsuccessful. In fact, one of the native crystals soaked in 10 mM GTP diffracted to $2.8 \AA$, but no GTP could be seen in the electron density map (Data not shown). This seems to suggest that either MoaC2 does not bind GTP or it binds rather weakly. Therefore to identify the ligand of MoaC2, In-silico docking studies were done with GTP and other possible intermediates compounds, in the formation of precursor Z as given by Kanaujia et al., 2010 [8]. Such structure based computational calculations provide significant mechanistic insights. Ongoing experimental work in one of groups (AA) have been initiated based on the study.

To identify the most likely ligand that binds to MoaC2, all compounds in the pathway from GTP to precursor Z (GTP, FPT, PBT, PBM, E and precursor Z) were docked to MoaC2 using Autodock 3.0.5 and their interaction energies calculated using its empirical scoring function. The docking of GTP shows hydrogen bonds between the oxygens of phosphates to the active site residues Lys62, Asp137, Lys 140, Leu60 of A chain and His88 of B chain, in agreement with the T. thermophilus structure [8] (Figure 6). Table S2 lists the docking energies for these ligands and the hydrogen bonds between the protein with GTP and probable ligands. From S2, it is evident that PBM has the highest docking energy, followed by GTP. Apart from the hydrogen bonds observed between GTP and MoaC2, which are consistent with the crystal structure, PBM has an additional hydrogen bond between Lys 140 with oxygen of carbonyl group of the benzo moiety (N-O32, $2.93 \AA$ ). The hydrogen bond distances are consistently shorter in PBM approximately by $\sim 0.50 \AA$ as compared to GTP. Qualitatively, this suggests that PBM is a better, and therefore more likely ligand than GTP which is also in agreement with the existing hypothesis, where MoaA acts on GTP and MoaC involved in the subsequent step of precursor $\mathrm{Z}$ synthesis. The docking studies also suggest that of the three pathways proposed for precursorZ formation by Kanaujia et al., 


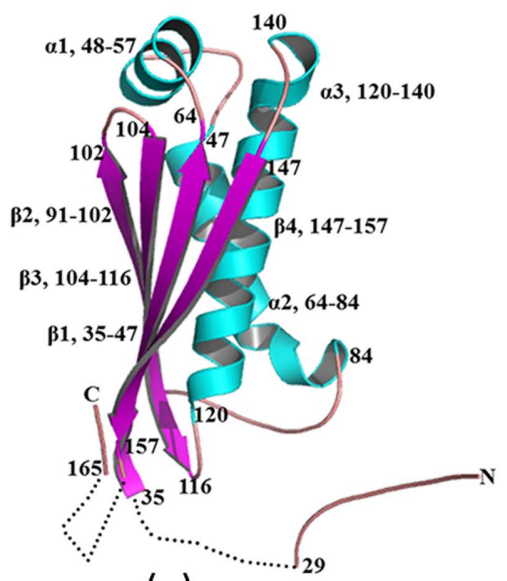

(a)

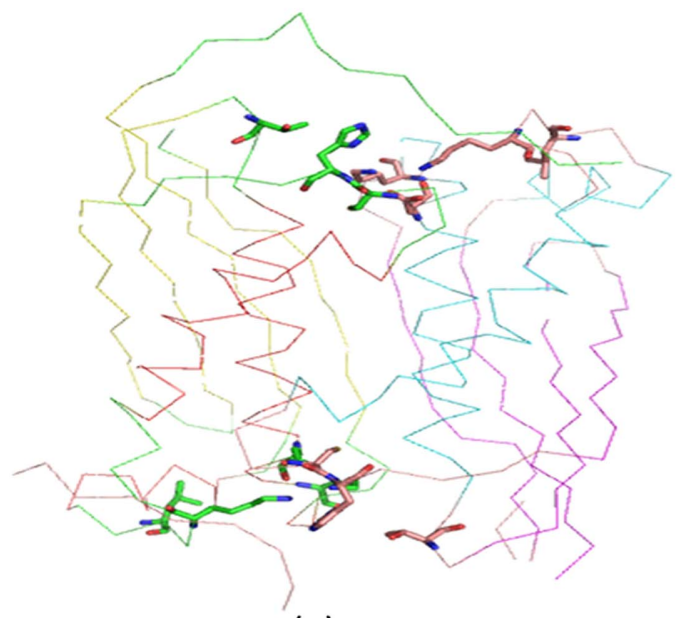

(c)
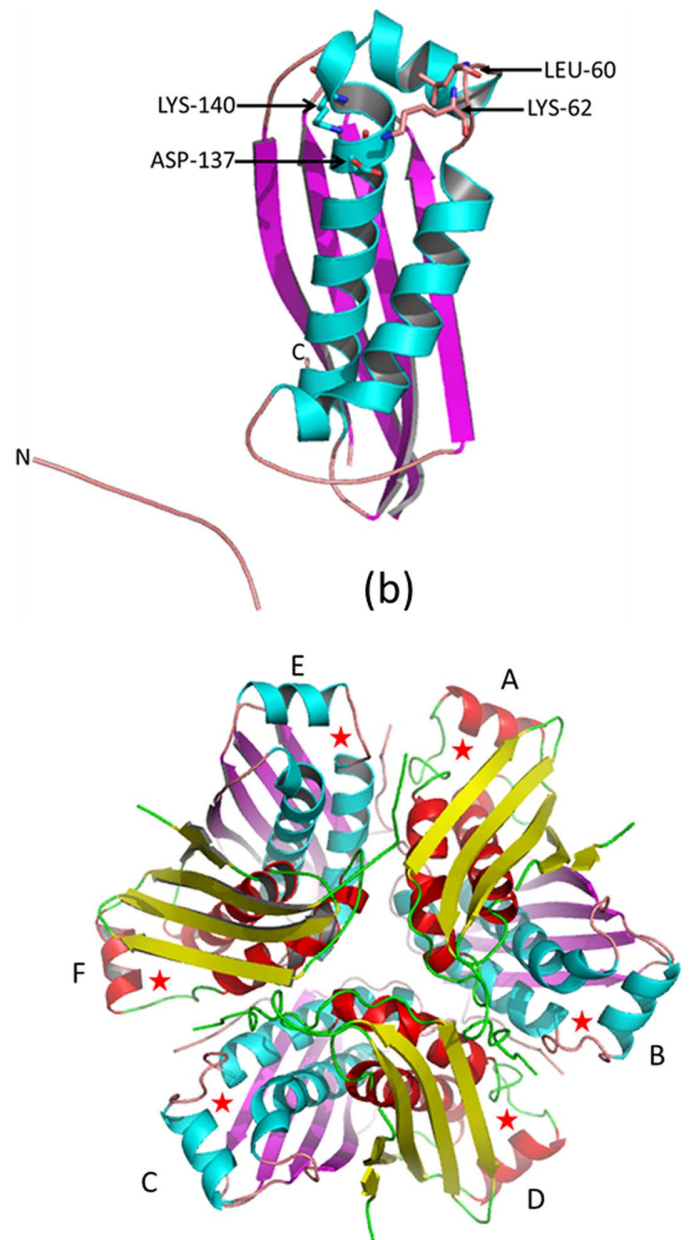

(d)

Figure 2. Tertiary and Quaternary structures of MoaC2 (Rv0864). Cartoon representation of the (a) monomer; helices are coloured in cyan and sheets in magenta and loop regions are in salmon. Missing regions are shown as dots. (b) Monomer showing the residues of the putative ligand binding site as sticks. (c) C- $\alpha$ trace of contents of the asymmetric unit (dimer) with the residues of the putative ligand binding sites shown as sticks.(d) hexamer the ligand binding site are shown as asterisks.

doi:10.1371/journal.pone.0058333.g002

one of them (Path 2b, Fig. 1) can be ruled out, based on comparative interaction energies for the different ligands (S2).

\section{Result of sequence based interaction study}

In order to find if moaA and moaC2 interact with each other during the formation of precursor $\mathrm{Z}$, the sequence of moaC2 was given as input to the STRING 9.0 (http://string-db.org/) [22] to identify potential interacting proteins and its result is shown in Figure 7 a. mog and two orthologous genes of moaA, moaAl $(\mathrm{Rv} 3109)$ and moaA2 (Rv0869c) are identified as potential interacting partners of MoaC2 ( $\mathrm{Rv0864}$ ). mog is annotated to be responsible for the downstream step of converting MPT to MPT-
AMP, while moaAl and moaA2 are involved in the same step of Moco biosynthesis. However, neither of the MoaA structures from M. tuberculosis is available in the PDB. BLAST searches of the sequences of both MoaAl and MoaA2, against the PDB were done, to identify homologous structures, showed a homologous structure from Staphylococcus aureus (PDB ID 2FB3) [7]. The $S$. aureus MoaA has a higher sequence identity with MoaAl (37.7\%) than MoaA2 (29\%). Hence, MoaAl was chosen as the representative MoaA structure for further in-silico Protein-Protein interaction studies, (Figure $7 \mathrm{~b}-\mathrm{d}$ ). As mentioned earlier, the SwissProt server (http://swissmodel.expasy.org/; [23] was used to build the model of moaAl.

Table 1. Buried surface area and hydrogen bonds at the intra-dimer and the inter-dimeric interfaces in hexamer .

\begin{tabular}{llll}
\hline Chains & No. of interface residues & Interface area $\AA^{\mathbf{2}}$ & No. of hydrogen bonds \\
\hline Intra-dimer & $35: 34$ & $1586: 1607$ & 14 \\
Inter-dimer & $14: 16$ & $727: 734$ & 8 \\
\hline doi:10.1371/journal.pone.0058333.t001 & &
\end{tabular}



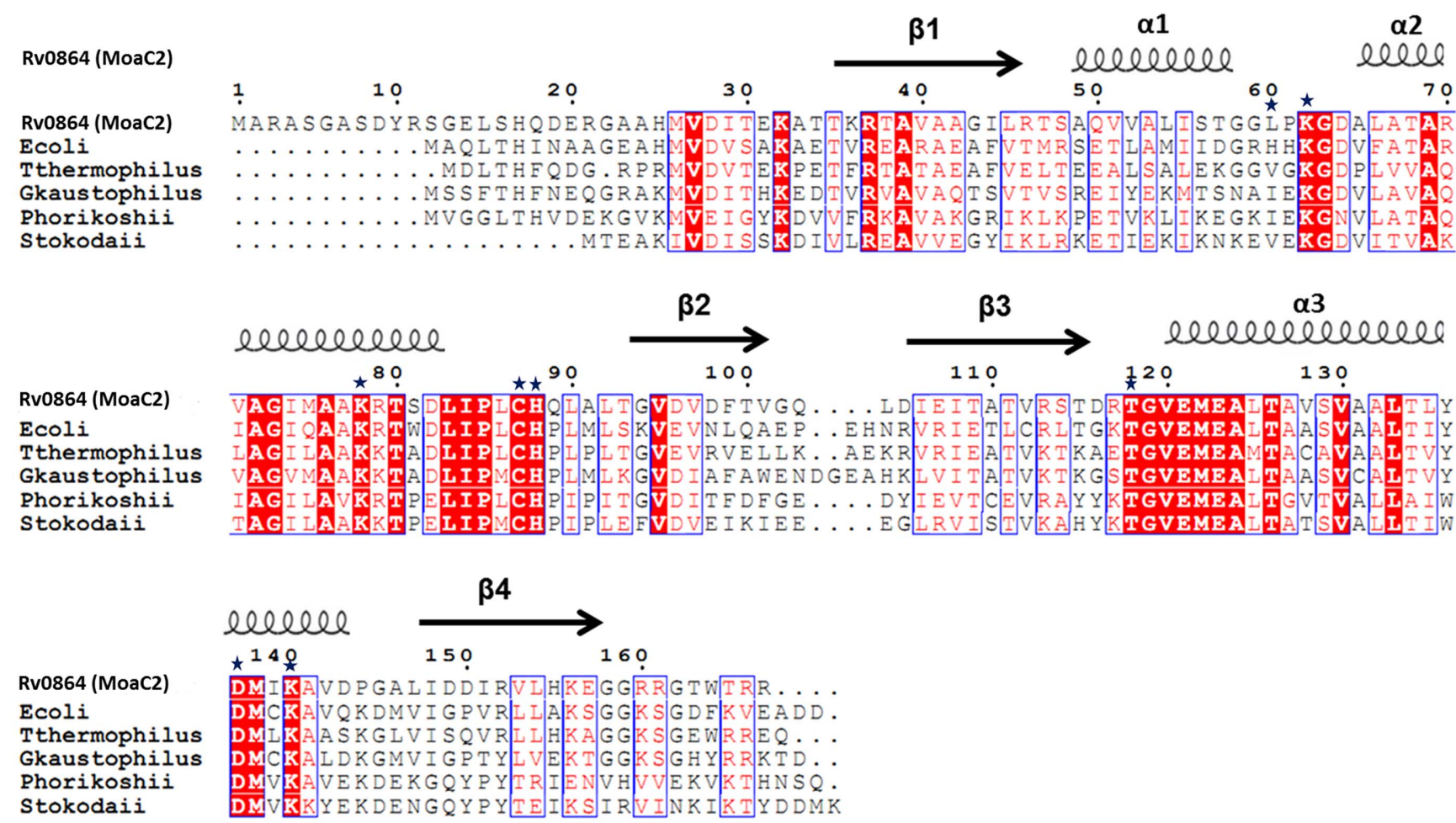

Figure 3. Structure based Sequence alignment of Rv0864 (MoaC2) with different MoaC proteins. Helices and strands are represented by coils and arrows respectively. Conserved residues are highlighted in red boxes. Residues of the ligand binding sites are marked with asterisks. The sequence alignment was produced using the program ClustalW [46] and the figure generated using ESPript 2.2 [47]. doi:10.1371/journal.pone.0058333.g003

Having identified that MoaAl and MoaC2 interact, the two sequences were then input to the PPI-Pred interaction server (http://bmbpcu36.leeds.ac.uk/ppi_pred/; [24] which identified and ranked three clusters of residues on both proteins as potential interacting sites. This further validates that MoaAl and MoaC2 may be interacting with each other.

\section{Result of protein-protein docking}

Current literature studies have not shown MoaC or MoaA to exist separately as monomers. The $S$. aureus MoaA too exists as a dimer. Therefore dimers of MoaC2 and MoaAl were given as input to the ClusPro 2.0 software. The content of the asymmetric unit of MoaC2 and the model of MoaAl were taken as the ligand and receptor respectively for docking studies and the output structures analyzed visually. Upon visual examination, approximately $2 / 3$ rds of the models show reasonable interaction between the dimers. Based on the interactions, most of these output models can be broadly grouped into a couple of modes. In the first mode that is seen in the majority of the models $(\sim 18)$, the interaction occurs mainly through the $\alpha 2$ helix and the subsequent loop of the first monomer and and the $\alpha \mathrm{l}$ helix) of the second monomer of MoaC2 with the C-terminus of both monomers of MoaAl, although one monomer is the dominant interacting partner with MoaC2. In another mode that is observed less frequently $(\sim 6)$, the dimeric interactions occurs mainly through the $\alpha 1$ helix, the loop residues $101-105$ and residues $142-150$ of the first monomer and Arg46, Glu103 and Asp150 of the second monomer (MoaC2) with the C-terminii of both the MoaAl monomers (Figure 8a). In this mode, most of the hydrogen bonds are formed between A monomer of MoaC2 with both monomers of MoaAl ( $\mathrm{X}$ and $\mathrm{Y}$ ). It has been reported in human homologs that C-terminal of MoaAl interacts with MoaC2 [15] and this is in qualitative agreement in both the modes of the docked model. Although this mode is found less frequently than the first, this mode is qualitatively better as both electrostatic and hydrophobic interactions contribute to this mode and it is also compatible with higher order oligomer (hexamer) whereas the other mode is predominantly electrostatic. Hence, this mode was considered for further analysis.

The interacting residues of the docked MoaC2 and MoaAl complex were identified by Protein Interactions calculator (PIC) web server (http://pic.mbu.iisc.ernet.in/; [25] as well by visually examining the docked models and are given in Table S3. The protein complex is stabilized by a variety of $\mathrm{H}$-bonds, ionic and hydrophobic interactions and is consistent with the predictions of PPI-Pred.

Having seen that the dimers of MoaAl and MoaC2 can form a complex, we went on to see if this docked model is compatible with other known oligomeric states. As mentioned earlier, the functional oligomeric state of both MoaA and MoaC are as yet unknown. While MoaAl has been shown to exist as a dimer in solution, MoaC2 can exist as a dimer, though it is most stable as a hexamer. This hexameric association is conserved in all the available structures of MoaC from different organisms, irrespective of the crystallization condition, crystal symmetry, etc. The docked MoaAl: MoaC2 model was then superposed on to the hexameric assembly of MoaC2 and is shown in Figure 8b. As can be seen, there are no steric clashes between the docked MoaAl dimer and the four other monomers of MoaC2.

Significantly, the docking has brought the ligand binding pockets of one monomer of MoaAl and MoaC2 in close proximity. The ligand binding region of the $\mathrm{X}$ monomer of MoaAl is approximately $17 \AA$ (as measured between the 


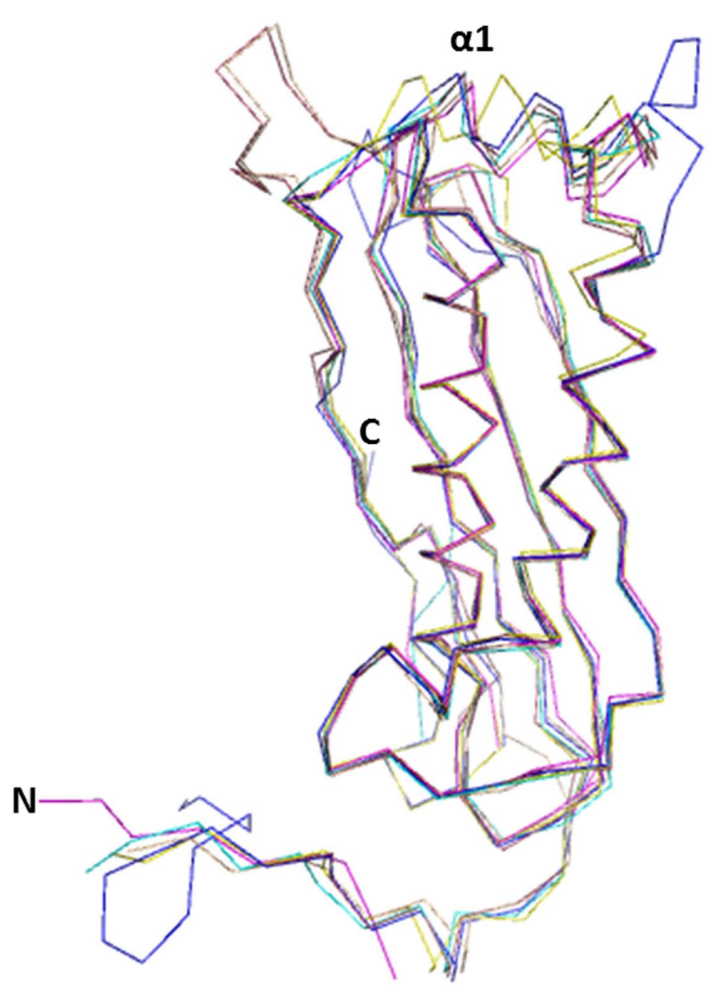

Figure 4. C- $\alpha$ trace of the structural superposition of the individual subunit of Rv0864 MoaC2 (magenta) with those of TtMoaC (yellow), EcMoaC (cyan), PhMoaC (wheat), StMoaC (raspberry) and GkMoaC (blue). The figure was generated using PyMOL (v.1.2r3pre; Schrodinger LLC).

doi:10.1371/journal.pone.0058333.g004 guanosine rings) from the ligand binding site of the A monomer of MoaC2. Figure 8c shows a surface representation of this region, with the ligands (GTP) superposed to their respective binding sites. As can be seen from Figure 8c, the product of the first enzyme (MoaAl) can access the adjacent binding site of MoaC2, to carry out the subsequent step in the Moco synthesis pathway. This apparent fusion of the two binding sites might also provide a basis that why GTP has the second highest docking score. Which clearly implies that why GTP was bound loosely in T. thermophilus structure. It is important to note that no information was provided to the docking server regarding the binding sites. In fact, both MoaAl and MoaC2 dimers were docked in their apo forms. Still, the apparent 'fusion' of the functional sites, as well as the compatibility with known oligomeric states of MoaC2, lends credence to the fact that these two proteins might indeed form a complex during precursor $\mathrm{Z}$ formation.

\section{Conclusions}

The crystal structure of the Moco-biosynthesis protein MoaC2 (Rv0864) in its apo form from Mycobacterium tuberculosis has been determined to $2.2 \AA$. The protein adopts a modified form of the ferredoxin fold, with an additional $\alpha$ helix. Analysis of this structure and comparison with the other homologs in the PDB show that 31 residues are conserved and these residues have a functional or a structural role.

Lys78, which is conserved in all the MoaC proteins is located in the $\alpha 2$ helix of the adjacent dimer form three strong hydrogen bond from its NZ atom (Average distance $2.8 \AA$ ) with the main chain carbonyl group of residues Ser81, Pro85 and Cys87 which results in an approximately three fold symmetrical arrangement. The $\alpha 1$ helix 48-57, which is not typically present in the ferredoxin fold, shows a higher deviation when superposed with the other existing reported structures and it is not typically present in ferredoxin like fold. This helix lies adjacent to the ligand

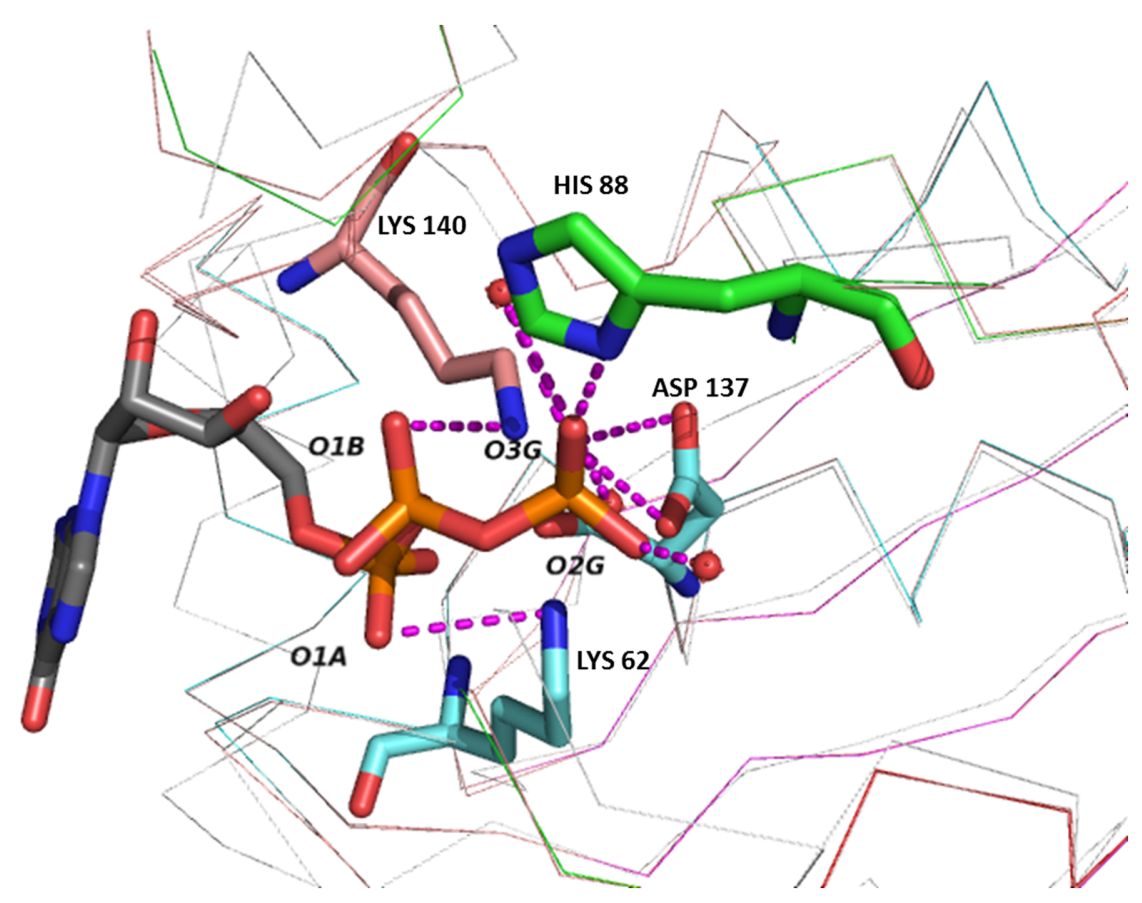

Figure 5. The putative ligand binding site of Rv0864 with GTP, obtained from its structural superposition on TtMoaC. Water molecules are represented as red spheres. The figure was generated using PyMOL (v.1.2r3pre; Schrodinger LLC). doi:10.1371/journal.pone.0058333.g005 


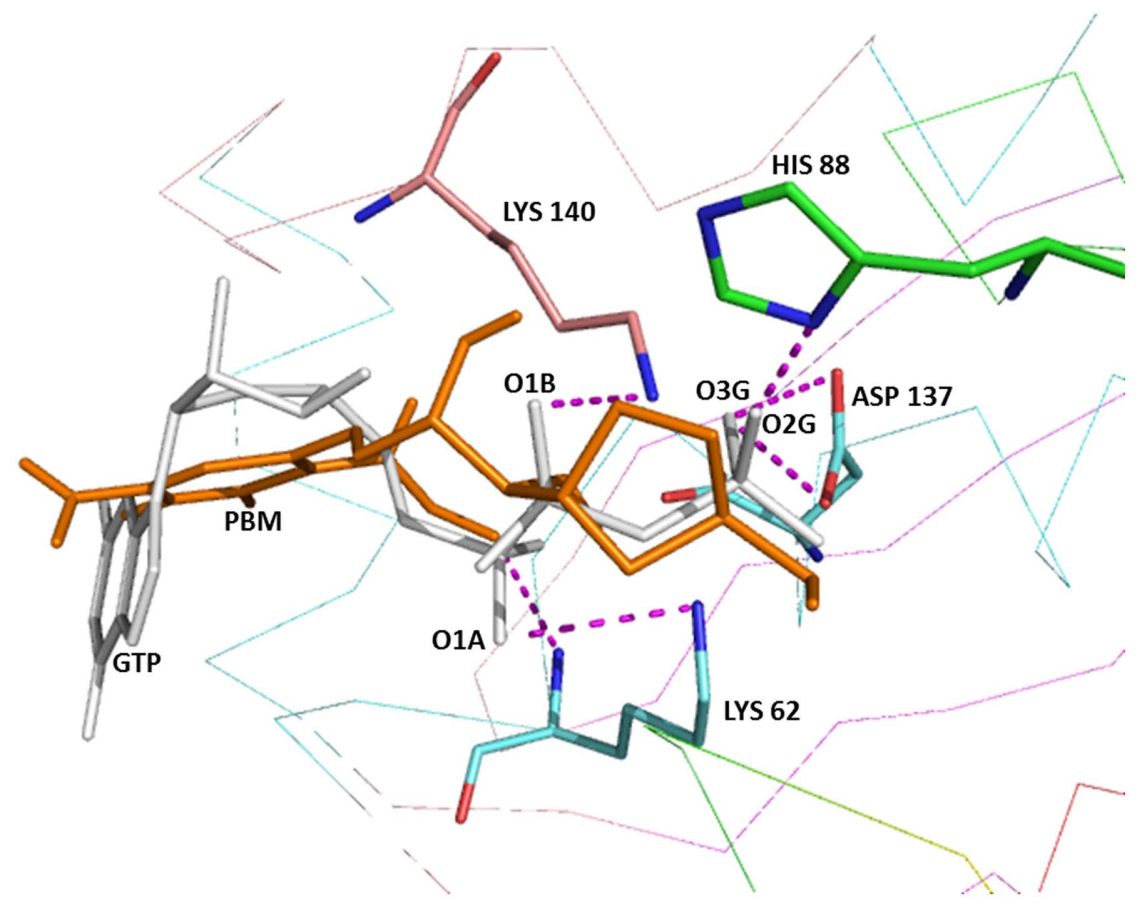

Figure 6. A close up view of the the ligand binding site of Rv0864 with the docked GTP and PBM, two of the possible ligands in the precursor $\mathbf{Z}$ formation pathway. Hydrogen bonds are represented as broken lines (magenta). doi:10.1371/journal.pone.0058333.g006

binding site and may impart functional role. Though the functional oligomeric state of the protein is still unknown, surface accessibility calculations suggest that it is stable both as a dimer and hexamer. This hexameric association is seen in available MoaC structures, suggesting that this association is important.

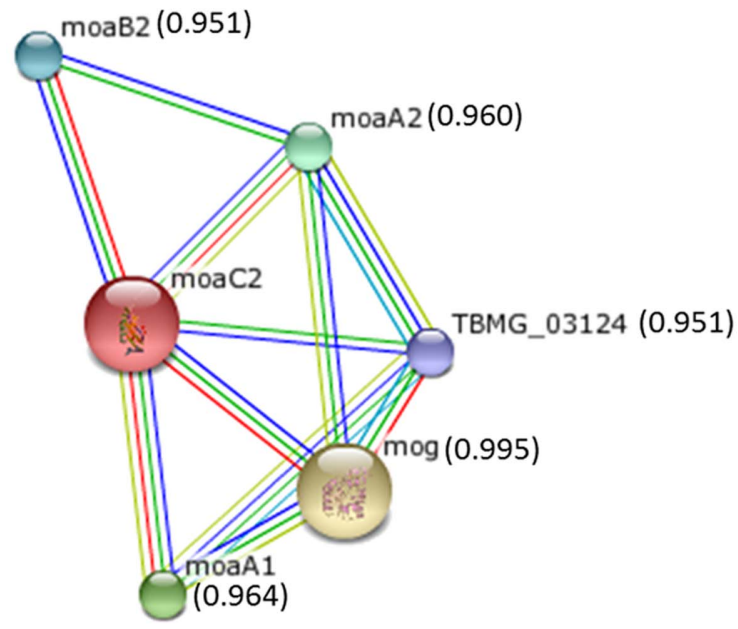

(a)

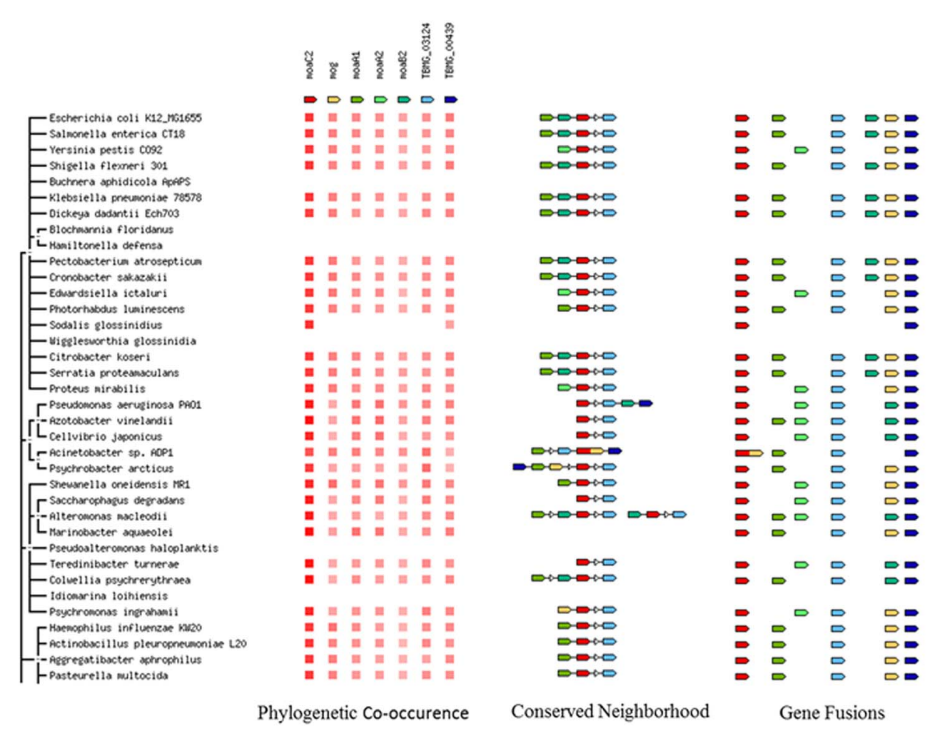

(b)

(c)

(d)

Figure 7. Summary of the outputs from String server with M. tuberculosis MoaC2 as the query molecule. (a) Network diagram connecting potential partner proteins. Green lines indicate association by recurring neighborhood, blue lines represent phylogenetic co-occurrence, red lines indicate gene-fusion events and light green corresponds to text mining; the thickness of each line is a rough indicator for the strength of the association. The interacting partners are mog (molybdopterin biosynthesis protein), moaA1 (molybdenum cofactor biosynthesis protein $\mathrm{A}$ moaA1), moaA2 (molybdenum cofactor biosynthesis protein A), moaB2 (pterin 4-alpha-carbinolamine dehydratase) and TBMG_03124 (molybdenum cofactor biosynthesis protein E2) and their confidence score are given in bracket. Contributions from (b) Phylogenetic co-occurrence in which a dark color shows the conservation of the gene and light color shows no homolog. (c) neighborhood wherein genes connected by lines are direct neighbors on the chromosome. (d) hybrid gene which is formed from two previously separate genes.

doi:10.1371/journal.pone.0058333.g007 


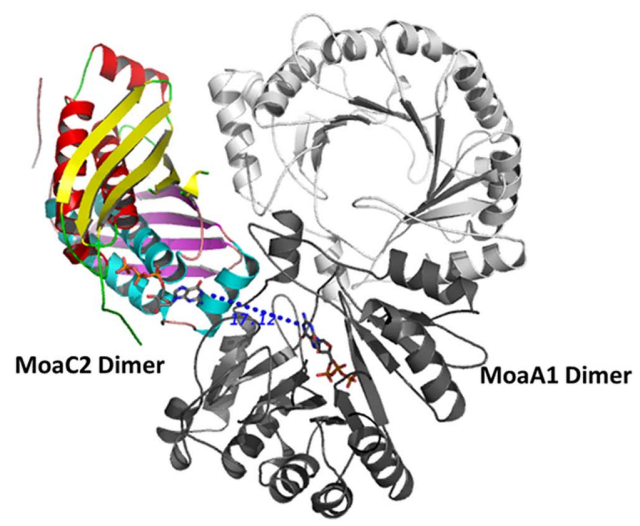

(a)
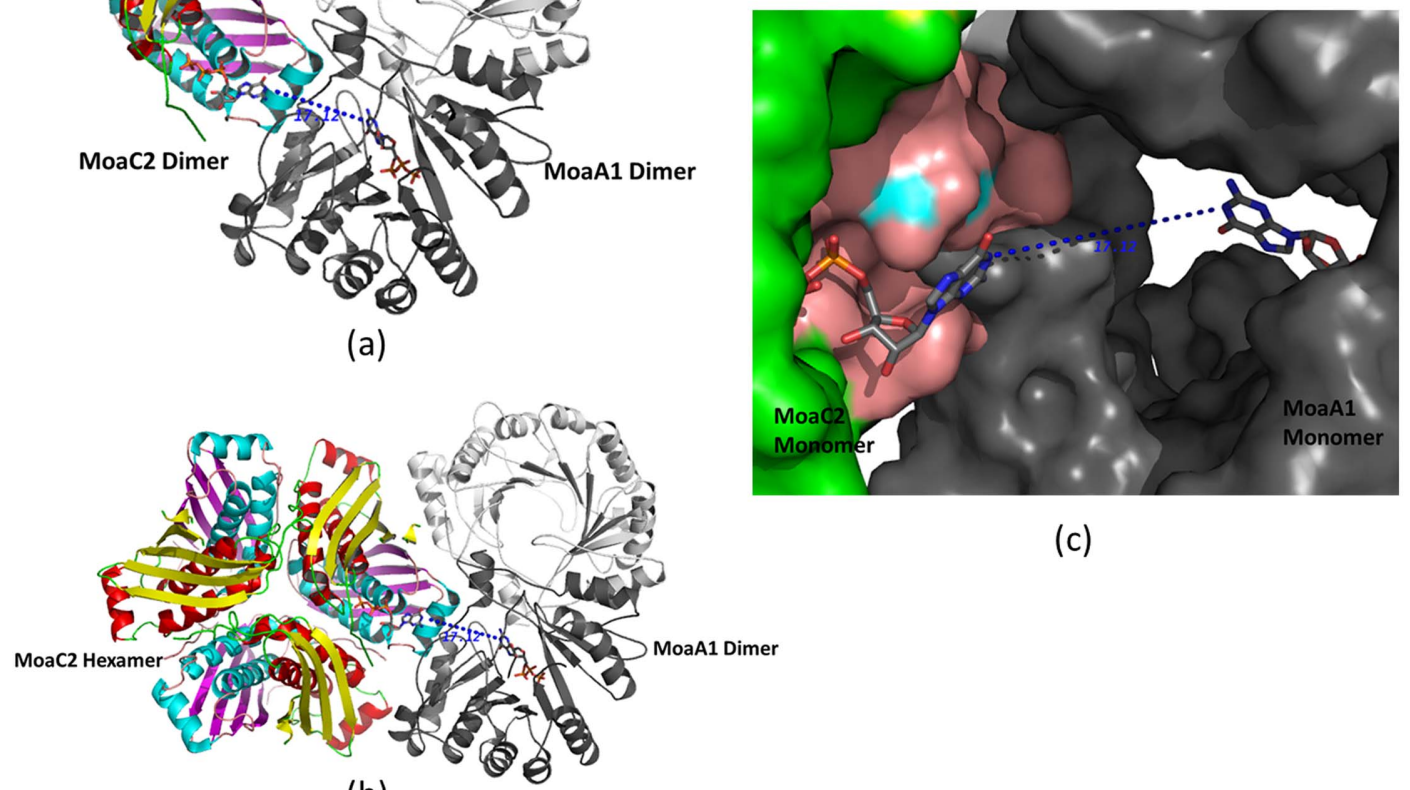

(c)

(b)

Figure 8. Schematic cartoon representation of the protein-protein docking, with the putative ligand sites identified by a GTP molecule represented as stick. Only the closest ligand sites are shown for clarity. (a) The docked model of MoaC2 dimer with MoaA1 dimer. (b) The docked model superposed on the MoaC2 hexamer. (c) A close up view in surface representation of the ligand binding sites of monomer A of MoaC2 and monomer X of MoaA1. The distance in $\AA$ is measured between the two guanosine rings.

doi:10.1371/journal.pone.0058333.g008

The structure of Rv0864 was solved in its apo form. Persistent efforts to crystallize the protein in the presence of GTP (cocrystallization as well as crystal soaking) did not result in a GTPbound structure. A review of literature suggested that two different pathways were plausible for the formation of precursor $\mathrm{Z}$ from GTP. The molecular-docking studies of the probable ligands with the protein suggested that PBM has the highest docking energy followed by GTP, suggesting PBM as the more likely ligand. PBM also has an additional H-bond, as compared to GTP. This study has also shown that one of the proposed pathways $(2 \mathrm{~b})$ can be eliminated as not energetically favourable.

The result of sequence based interaction study using STRING 9.0 helped in identifying the interacting partner of MoaC2. Once MoaAl was identified as the interacting partner, the Protein Protein Interaction-Pred server was used to predict the interacting residues. This server too confirmed the interaction between the two proteins and identified patches on both proteins as interacting residues. In the absence of structure of M.tuberculosis MoaAl, a model was built using a homologous structure from the PDB as a template. The two structures were then docked to better understand the interaction between MoaC2 and MoaAl. The interaction between dimers- is possible, as dimers of both MoaC2 and MoaAl are stable in solution [15]. The dimer-dimer interaction between MoaC2 and MoaAl occurs mainly through a helix and a loop of MoaC2 with the C-terminal of both monomers of MoaAl. More importantly, the docking has resulted in creating a channel from the binding sites of MoaAl and MoaC2 that is conducive for the transfer of the product generated by
MoaAl to become the substrate for MoaC2. Further experimental work has been initiated in one (AA) of our laboratories.

\section{Materials and Methods}

\section{Data collection and data statistics}

The cloning, overexpression, purification, crystallization, data collection and structure solution of the protein has been described previously [3]. While refinement of this structure at $2.5 \AA$ was in progress, another data set from a crystal grown from an identical crystallization condition as before was collected at Beamline BM14, ESRF, Grenoble. This crystal diffracted to $2.20 \AA$ with an exposure time of $11 \mathrm{sec}$, the crystal to detector distance of $207 \mathrm{~mm}$ and an oscillation range of $0.5^{\circ}$. The reflections were indexed using the iMOSFLM program [26] and scaled with SCALA [27]. The CTRUNCATE program [28] was used to convert intensities to structure factors. HKL1.98.2 [29] suite was also used for processing and scaling of the data sets. POINTLESS [27] suggests the possible space group for this dataset as $P 2_{1} 3$, similar to the earlier data. The only difference between the two data sets is the reduction in the cell length by $2.6 \AA$. The diffraction data statistics are given in Table 2.

\section{Structure solution, Refinement and validation}

As the cell dimensions between the two datasets differ by $\sim 3 \%$, the previously refined partial model was taken as the search model for, molecular-replacement calculations, done using Phaser [30] as implemented in the CCP4 suite [31] which resulted in a unique solution with no steric clashes. Two copies of the search model 
Table 2. Data Collection Statistics.

\begin{tabular}{|c|c|}
\hline Wavelength $(\AA)$ & 0.97 \\
\hline Space Group & $\mathrm{P} 2,3$ \\
\hline Unit cell parameter $(\AA ̊)$ & 91.9 \\
\hline Resolution range $(\AA)$ & $27.7-2.20(2.28-2.20)$ \\
\hline No. of observations & 75559 (8529) \\
\hline No. of unique reflection & $12651(1550)$ \\
\hline Multiplicity & $5.9(5.5)$ \\
\hline $\mathrm{I} / \sigma(\mathrm{I})$ & $9(2.5)$ \\
\hline Completeness (\%) & $99.9(99.9)$ \\
\hline$R_{\text {merge }}^{\dagger}$ & $0.17(58)$ \\
\hline \multicolumn{2}{|l|}{ Refinement statistics } \\
\hline$R_{\text {work }}$ & 0.20 \\
\hline$R_{\text {free }}$ & 0.24 \\
\hline \multicolumn{2}{|c|}{ Deviation from ideal geometry } \\
\hline Bond length $(\AA)$ & 0.009 \\
\hline Bond angle $\left({ }^{\circ}\right)$ & 1.11 \\
\hline \multicolumn{2}{|l|}{ Protein Model } \\
\hline No. of subunits in A.U & 2 \\
\hline Protein Atoms & 1922 \\
\hline Water Molecules & 65 \\
\hline \multicolumn{2}{|l|}{ Ramachandran plot (\%) } \\
\hline Mostly favoured regions & 92.1 \\
\hline Additional allowed regions & 7.1 \\
\hline Generously allowed regions & 0.4 \\
\hline Disallowed regions & 0.4 \\
\hline \multicolumn{2}{|c|}{$\begin{array}{l}{ }^{\dagger} R_{\text {merge }}=\Sigma_{\mathrm{hkl}} \Sigma_{\mathrm{i}} \mathrm{l}_{\mathrm{i}}(\mathrm{hkl})-(\mathrm{l}(\mathrm{hkl})) \mid / \Sigma_{\mathrm{hkl}} \Sigma_{\mathrm{i}} \mathrm{l}_{\mathrm{i}}(\mathrm{hkl}) ; \text { where } \mathrm{l}_{\mathrm{i}}(\mathrm{hkl}) \text { is the intensity of the } \\
\text { ith observation of reflection } \mathrm{hkl} \text { and }(\mathrm{l}(\mathrm{hkl})) \text { is the average intensity of the } \mathrm{i} \\
\text { observations. Values in parentheses are for the highest resolution shell. } \\
\text { doi:10.1371/journal.pone.0058333.t002 }\end{array}$} \\
\hline
\end{tabular}

were unambiguously placed in the asymmetric unit. The solution obtained from the MR calculation was subjected to rigid-body refinement using PHENIX [32] and subsequently positional refinement was performed. The crystallographic $R$ factor and $R_{\text {free [33] converged to values of } 27 \% \text { and } 33 \% \text { respectively and }}$ electron density maps computed. After several rounds of model building using Coot [34] and refinement with REFMAC5 from $C C P 4$ suite, the $R$ factor and $R_{\text {free }}$ values converged to $20 \%$ and $24 \%$ respectively. To remove any model bias a simulated annealing omit map [32] was calculated and the resultant electron density map agreed with the model. All atoms were refined with unit occupancies. In the final refined model, the A chain, lacks 21 residues at the N-terminus as well as residues 30-34, 158-164, while in the $\mathrm{B}$ chain there is no interpretable electron density for the N-terminal 22 residues and residues 158-162 near the Gterminus. The final model also accommodates 65 water molecules. All the residues are in the allowed regions of the Ramachandran map [35] except one residue at the $\mathrm{N}$ - and C-termini of the A chain (Ala23 and Arg166) and other geometric parameters are also well within the acceptable values. The program PROCHECK [36] and MOIPROBITY [37] were used to check and validate the quality of the final refined models. Co-ordinates have been deposited in the Protein Data Bank [38] with accession code $4 \mathrm{FDF}$.
Docking studies with GTP and intermediates compound formed during the formation of precursor $Z$

Docking studies were performed with GTP and other probable ligands as mentioned in Kanaujia et al., 2010 [8] in order to estimate their binding affinities towards MoaC2 (Rv0864) using the program AUTODOCK 3.0.5 [39]. The Lamarckian genetic algorithm implemented in Autodock is a hybrid of a genetic algorithm (GA) with an adaptive local search (LS) method [40].

The 3D structures of the ligands GTP, FPT, PBT, PBM and E were built and optimized using the BUILDER module in Insight II [41] and Sybyl 8.0 (Tripos Associates, Inc., St. Louis, MO). After docking, the interaction energy between the docked ligand and the receptor protein was calculated using the empirical scoring function feature of Autodock. Rests of the parameters were set to their default values.

\section{Sequence based interaction studies}

In order to identify the interaction of Rv0864 (MoaC2) with other interacting partner the sequence based interaction database STRING (http://string-db.org/) was used. The software runs a set of prediction algorithms and transfers known interactions from model organisms to other species based on predicted orthology of the corresponding proteins [42,43]. The software identifies potential interacting partners based on a varied set of diverse interactions, including existing information from other organisms, genetics, phylogenetic co-occurences, functional associations and the output is given in the form of a confidence score for each of the four methods.

\section{Protein-Protein Docking}

Having identified that MoaAl and MoaC2 can interact directly, the two proteins were docked computationally using the ClusPro 2.0 server [44]. Mycobacterium tuberculosis MoaAl is 359 amino acid long and its structure is unknown. However, a BLAST search of the MoaAl sequence against the PDB identified a homologue from Staphylococcus aureus (PDBID 2FB3) [7], with a sequence identity of $37 \%$. A homology model of MoaAl was generated with the $S$. aureus model as the template using the SwissProt server and validated using standard tools.

To predict the structure of a complex, Cluspro 2.0 requires only the atomic coordinates of the two molecules and outputs forty docked models based on electrostatic, hydrophobic, Van der Wallelectrostatic and balanced interactions (10 docked conformations for each type of interaction). Although the models are ranked by a scoring function, the authors recommend that these solutions be analysed visually to decide its feasibility. The interactions observed in these docked conformations were visually examined using the software PyMol (v.1.2r3pre; Schrodinger LLG) and also using the Protein Protein Interface Prediction Server PPI-Pred (http:// bmbpcu36.leeds.ac.uk/ppi_pred/; [24] and PIC web server (http://pic.mbu.iisc.ernet.in/;) [25].

\section{Structural analysis}

Structures were superposed using the program STAMP [45]. It uses multiple sequence alignment using the amino acid sequence information followed by an initial superposition of structures. Accessible surface area, number of contacts and temperature factor was calculated using the program Areaimol, Contact and Baverage [31]. The sequence alignment was produced using the program ClustalW [46] and the figure was generated using the program ESPript 2.2 [47]. The PISA web server (http://www.ebi.ac. uk/msd-srv/prot_int/cgi-bin/piserver; [19] was used to know the 
biological assembly and to calculate accessible surface area and buried surface area of protein.

\section{Supporting Information}

Table $S 1$ Root mean square deviation in $\alpha 1$ region of Rv0864 (MoaC2) with other structure.

(DOG)

Table S2 List of hydrogen bonds between source atom (MoaC2) and different ligands were given and docking energy of different ligands with Rv0864 (MoaC2) was given in $\mathrm{kcal} / \mathrm{mol}$.

(DOC)

\section{References}

1. Wuebbens MM, Rajagopalan KV (1995) Investigation of the early steps of molybdopterin biosynthesis in Escherichia coli through the use of in vivo labeling studies. The Journal of biological chemistry 270: 1082-1087.

2. Rieder G, Eisenreich W, O'Brien J, Richter G, Gotze E, et al. (1998) Rearrangement reactions in the biosynthesis of molybdopterin-an NMR study with multiply $13 \mathrm{C} / 15 \mathrm{~N}$ labelled precursors. European journal of biochemistry 255: $24-36$.

3. Srivastava S, Srivastava VK, Arora A, Pratap JV(2012) Overexpression, purification,crystallization and preliminary $\mathrm{X}$-ray analysis of putative molybdenum cofactor biosynthesis protein $\mathrm{C}$ (MoaC2) from Mycobacterium tuberculosis H37Rv. Acta Crystallogr Sect F Struct Biol Cryst Commun 68: 687-691.

4. Frey PA, Magnusson OT (2003) S-Adenosylmethionine: a wolf in sheep's clothing, or a rich man's adenosylcobalamin? Chemical reviews 103: 21292148 .

5. Jarrett JT (2003) The generation of 5'-deoxyadenosyl radicals by adenosylmethionine-dependent radical enzymes. Current opinion in chemical biology 7: 174-182.

6. Sofia HJ, Chen G, Hetzler BG, Reyes-Spindola JF, Miller NE (2001) Radical SAM, a novel protein superfamily linking unresolved steps in familiar biosynthetic pathways with radical mechanisms: functional characterization using new analysis and information visualization methods. Nucleic acids research 29: 1097-1106.

7. Hanzelmann P, Schindelin $\mathrm{H}$ (2006) Binding of $5^{\prime}$-GTP to the C-terminal FeS cluster of the radical S-adenosylmethionine enzyme MoaA provides insights into its mechanism. Proceedings of the National Academy of Sciences of the United States of America 103: 6829-6834.

8. Kanaujia SP, Jeyakanthan J, Nakagawa N, Balasubramaniam S, Shinkai A, et al. (2010) Structures of apo and GTP-bound molybdenum cofactor biosynthesis protein MoaC from Thermus thermophilus HB8. Acta crystallographica 66: 821-833.

9. Reiss J, Cohen N, Dorche C, Mandel H, Mendel RR, et al. (1998) Mutations in a polycistronic nuclear gene associated with molybdenum cofactor deficiency. Nature genetics 20: 51-53.

10. Hoff T, Schnorr KM, Meyer C, Caboche M (1995) Isolation of two Arabidopsis cDNAs involved in early steps of molybdenum cofactor biosynthesis by functional complementation of Escherichia coli mutants. The Journal of biological chemistry 270: 6100-6107.

11. Unkles SE, Smith J, Kanan GJ, Millar LJ, Heck IS, et al. (1997) The Aspergillus nidulans cnxABC locus is a single gene encoding two catalytic domains required for synthesis of precursor $\mathrm{Z}$, an intermediate in molybdenum cofactor biosynthesis. The Journal of biological chemistry 272: 28381-28390.

12. Hanzelmann P, Schwarz G, Mendel RR (2002) Functionality of alternative splice forms of the first enzymes involved in human molybdenum cofactor biosynthesis. The Journal of biological chemistry 277: 18303-18312.

13. Rudolph MJ, Wuebbens MM, Rajagopalan KV, Schindelin H (2001) Crystal structure of molybdopterin synthase and its evolutionary relationship to ubiquitin activation. Nature structural biology 8: 42-46.

14. Leimkuhler S, Wuebbens MM, Rajagopalan KV (2001) Characterization of Escherichia coli MoeB and its involvement in the activation of molybdopterin synthase for the biosynthesis of the molybdenum cofactor. The Journal of biological chemistry 276: 34695-34701.

15. Wuebbens MM, Liu MT, Rajagopalan K, Schindelin H (2000) Insights into molybdenum cofactor deficiency provided by the crystal structure of the molybdenum cofactor biosynthesis protein MoaC. Structure 8: 709-718.

16. Betts JC, Lukey PT, Robb LC, McAdam RA, Duncan K (2002) Evaluation of a nutrient starvation model of Mycobacterium tuberculosis persistence by gene and protein expression profiling. Molecular microbiology 43: 717-731.

17. Wuebbens MM, Rajagopalan KV (1993) Structural characterization of a molybdopterin precursor. The Journal of biological chemistry 268: 1349313498.
Table S3 Hydrogen bonding in between MoaC2 and MoaA1 of Model 2.

(DOG)

\section{Acknowledgments}

JVP and VKS thank DBT-Wellcome Trust for access to beamline BM14 at the ESRF, Grenoble and to the beamline staff. This manuscript bears the GDRI communication No. 8395.

\section{Author Contributions}

Conceived and designed the experiments: AA JVP VKS. Performed the experiments: VKS SS. Analyzed the data: VKS JVP. Contributed reagents/materials/analysis tools: AA JVP. Wrote the paper: VKS SS AA JVP.

18. Dutta NK, Mehra S, Didier PJ, Roy CJ, Doyle LA, et al. (2010) Genetic Requirements for the Survival of Tubercle Bacilli in Primates. The Journal of infectious disease 201: 1743-1752.

19. Krissinel E, Henrick K (2007) Inference of macromolecular assemblies from crystalline state. Journal of molecular biology 372: 774-797.

20. Yoshida H, Yamada M, Kuramitsu S, Kamitori S (2008) Structure of a putative molybdenum-cofactor biosynthesis protein $\mathrm{C}(\mathrm{MoaC})$ from Sulfolobus tokodaii (ST0472). Acta Crystallogr Sect F Struct Biol Cryst Commun 64: 589-592.

21. Hanzelmann P, Schindelin H (2004) Crystal structure of the S-adenosylmethionine-dependent enzyme MoaA and its implications for molybdenum cofactor deficiency in humans. Proceedings of the National Academy of Sciences of the United States of America 101: 12870-12875.

22. Szklarczyk D, Franceschini A, Kuhn M, Simonovic M, Roth A, et al. (2011) The STRING database : functional interaction networks of proteins, globally integrated and scored. Nucleic acids research 39: D561-568.

23. Arnold K, Bordoli L, Kopp J, Schwede T (2006) The SWISS-MODEL workspace: a web-based environment for protein structure homology modelling. Bioinformatics (Oxford, England) 22: 195-201.

24. Bradford JR, Westhead DR (2005) Improved prediction of protein-protein binding sites using a support vector machines approach. Bioinformatics (Oxford, England) 21: 1487-1494

25. Tina KG, Bhadra R, Srinivasan N (2007) PIC: Protein Interactions Calculator. Nucleic acids research 35: W473-476.

26. Battye TG, Kontogiannis L, Johnson O, Powell HR, Leslie AG (2011) iMOSFLM: a new graphical interface for diffraction-image processing with MOSFLM. Acta crystallographica D 67: 271-281.

27. Evans $\mathrm{P}$ (2006) Scaling and assessment of data quality. Acta crystallographica 62: 72-82.

28. French S, Wilson K (1978) On the treatment of negative intensity observations. Acta Cryst A 34: 517-525.

29. Otwinowski Z, Minor W (1997) Processing of X-ray Diffraction Data Collected in Oscillation Mode. Methods in Enzymology 276: 307-326.

30. McCoy AJ, Grosse-Kunstleve RW, Adams PD, Winn MD, Storoni LC, et al. (2007) Phaser crystallographic software. J Appl Cryst 40: 658-674.

31. Winn MD, Ballard CC, Cowtan KD, Dodson EJ, Emsley P, et al. (2011) Overview of the CCP4 suite and current developments. Acta Cryst 67: 235-242.

32. Adams PD, Afonine PV, Bunkóczi G, Chen VB, Davis IW, et al. (2010) Acta Crystallogr D 66: 213-221.

33. Brunger AT (1992) Free R value: a novel statistical quantity for assessing the accuracy of crystal structures. Nature 355: 472-475.

34. Emsley P, Cowtan K (2004) Coot: model-building tools for molecular graphics. Acta crystallographica 60: 2126-2132.

35. Ramachandran GN, Sasisekharan V (1968) Conformation of polypeptides and proteins. Advances in protein chemistry 23: 283-438.

36. Laskowski RA, MacArthur MW, Moss DS, Thornton JM (1993) A program to check the stereochemical quality of protein structures. J Appl Cryst 26: 283-291.

37. Chen VB, Arendall WB 3rd, Headd JJ, Keedy DA, Immormino RM, et al. (2010) MolProbity: all-atom structure validation for macromolecular crystallography. Acta crystallographica D 66: 12-21.

38. Berman HM, Westbrook J, Feng Z, Gilliland G, Bhat TN, et al. (2000) The Protein Data Bank. Nucleic acids research 28: 235-242.

39. Goodsell DS, Morris GM, Olson AJ (1996) Automated docking of flexible ligands:applications of AutoDock. J Mol Recognit 9: 1-5.

40. Morris GM, Goodsell DS, Halliday RS, Huey R, Hart WE, et al. (1998) Automated Docking Using a Lamarckian Genetic Algorithm and Empirical Binding Free Energy Function. J Computational Chemistry 19: 1639-1662.

41. ACCELRYS ver. 1.1. San Diego CA Accelrys (2000). 92121-92152.

42. von Mering C, Huynen M, Jaeggi D, Schmidt S, Bork P, et al. (2003) STRING: a database of predicted functional associations between proteins. Nucleic acids research 31: 258-261. 
43. von Mering C, Jensen LJ, Snel B, Hooper SD, Krupp M, et al. (2005) STRING: known and predicted protein-protein associations, integrated and transferred across organisms. Nucleic acids research 33: D433-437.

44. Comeau SR, Kozakov D, Brenke R, Shen Y, Beglov D, et al. (2007) ClusPro: performance in CAPRI rounds 6-11 and the new server. Proteins 69: 781-785.

45. Russell RB, Barton GJ (1992) Multiple protein sequence alignment from tertiary structure comparison: assignment of global and residue confidence levels. Proteins 14: 309-323.
46. Thomson JD, Higgins DG, Gibson TJ (1994) ClustalW : improving the sensitivity of progressive multiple sequence alignment through sequence weighting, position specific gap penalties and weight matrix choice. Nucleic Acids Res 22(22):4673-80.

47. Gouet P, Courcelle E, Stuart DI, Metoz F (1999) ESPript: analysis of multiple sequence alignments in PostScript. Bioinformatics 15: 305-308. 